\section{Notfallambulanz: Ketamin zusätzlich zu Morphin steigert die Effektivität}

\author{
Der Opioidverbrauch ist auch in US-Notfallambulanzen deutlich gestiegen. \\ Viel wird derzeit unternommen, um den hohen Verbrauch wieder zu reduzie- \\ ren. Eine monozentrische Studie untersuchte den Effekt von morphin- \\ adjuvantem Ketamin.
}

$\mathrm{n}$ den USA hat der Opioidverbrauch in den Notfallambulanzen zugenommen. Rund $30 \%$ der Patienten werden dort mit Opioiden versorgt, vor 15 Jahren waren es $20 \%$. Der Einsatz wird kontrovers diskutiert, verwiesen wird einerseits auf klinische Erfahrungen und Tradition, anderseits haben mit zunehmender Opioidverordnungsrate auch die Todesfälle durch Überdosierungen zugenommen. Eine alternative Möglichkeit, die Schmerzen zu lindern, bieten subdissoziative Dosen des N-methyl-D-aspartat (NMDA)-Rezeptorantagonisten Ketamin. Dessen analgetisierender Effekt in Dosen von unter $1 \mathrm{mg} / \mathrm{kg}$ i.v. wurde bereits bei Patienten mit Neuropathien und krebsbedingten Schmerzen dokumentiert. Dass Ketamin auch bei
Schmerzpatienten in Notfallambulanzen nützlich sein kann, haben kleine Studien ebenfalls schon gezeigt. In einer prospektiven monozentrischen Studie wurde das Applikationsschema etwas verändert und der Effekt erneut überprüft.

So erhielten 60 Patienten mit mäßigen bis starken akuten Schmerzen randomisiert und doppelblind neben $0,1 \mathrm{mg} / \mathrm{kg}$ Morphin i.v. entweder 0,3 mg/kg Ketamin als 15-min-Infusion oder Placebo. Primäres Studienziel war die Schmerzintensität auf einer 10-Punkte-Skala 15 Minuten nach Beginn der Intervention. Eines der sekundären Studienziele war die Zufriedenheit der Patienten mit dem Notfall-Schmerzmanagement, gemessen anhand einer 10-Punkte-Likert-Skala.
Nach 15 Minuten gaben die Patienten der Ketamingruppe im Median eine signifikant geringere Schmerzintensität an als die Patienten der Kontrollgruppe (3,5 [Interquartilsabstand: IQR 1,0 - 7,3] vs. $6,0[\mathrm{IQR} 4,0-9,0], \mathrm{p}=0,018$ ). Patienten mit zusätzlicher Ketamin-Medikation waren außerdem mit der Schmerzbehandlung zufriedener als die Kontrollpatienten $(8,57$ [Standardabweichung: SD 2,1] vs. 6,05 [SD 2,6], $p=0,01)$. Kein Unterschied zwischen den Gruppen bestand bei der zusätzlichen Bedarfsmedikation und der Länge des Ambulanzaufenthalts, schwere Nebenwirkungen wurden nicht berichtet.

Fazit: Subdissoziative Dosen von Ketamin, zusätzlich zu Morphin als 15-Minuten-Kurzinfusion gegeben, lindern mäßige bis starke Akutschmerzen bei Notfallambulanzpatienten besser als Morphin alleine. Der Effekt ist bereits nach 15 Minuten signifikant.

\section{Dr. Barbara Kreutzkamp}

Sin Billy et al. The use of ketamine for acute treatment of pain: a randomized, double-blind, placebo-controlled trial. J Emerg Med 2017; 52:601-8

\section{Keine Evidenz für Gabapentinoide bei unspezifischem Kreuzschmerz}

\begin{abstract}
Der Einsatz von Gabapentin und Pregabalin bei chronischer unspezifischer Lumbalgie wird durch die wenigen Studien in dieser Indikation nicht gestützt.
\end{abstract}

G abapentinoide sind wirksame Medikamente zur Linderung neuropathischer Schmerzen. Sie werden aber zunehmend auch gegen chronische Kreuzschmerzen unklarer Herkunft eingesetzt. Dieser Off-label-Gebrauch lässt sich mit den vorhandenen Studien nicht rechtfertigen, wie nun eine Metaanalyse zeigt.

Bislang sind laut den Ärzten um Harsha Shanthanna nur acht randomisierte kontrollierte Studien zum Einsatz von Gabapentinoiden bei mehr als drei Monate anhaltenden Kreuzschmerzen veröffentlicht worden. Die gemeinsame Auswertung der drei Studien zu Gabapentin mit zusammen 185 Patienten ergab im Placebovergleich lediglich eine minimale Schmerzreduktion, die mittlere Differenz auf einer Skala von 0-10 betrug nur 0,22 Einheiten. Die Qualität der Evidenz wurde mit dem GRADESystem als "sehr niedrig“ eingeschätzt.

Die Pregabalinmonotherapie schnitt in drei Studien mit insgesamt 332 Patienten weniger gut ab als die Vergleichssubstanzen (Amitriptylin, Celecoxib oder Tramadol/Paracetamol): Der mittlere Unterschied zugunsten der anderen Wirkstoffe betrug 0,42 Einheiten, bei ebenfalls sehr geringer Evidenzstärke.

In der größten Studie mit 181 Patienten machte es hinsichtlich der Schmerzlinderung keinen Unterschied, ob die Patienten Tapentadol allein oder in
Kombination mit Pregabalin erhielten. Nur in den beiden kleineren Studien, mit 44 und 36 Teilnehmern, hatte die Pregabalinzugabe einen deutlichen Nutzen gegenüber der alleinigen Behandlung mit Buprenorphin oder Celecoxib.

Bei sekundären Endpunkten wie funktionellen und emotionalen Verbesserungen, zeigten sich ebenfalls keine Unterschiede zu den Vergleichsgruppen.

Fazit der Autoren: Die bisherige Datenlage spricht nicht für den Einsatz von Gabapentinoiden bei überwiegend chronischen unspezifischen Kreuzschmerzen, lautet das Fazit von Shanthanna und Kollegen. Um den Nutzen der Substanzklasse abschließend beurteilen zu können, seien große und methodisch bessere Studien notwendig.

Dr. Beate Schumacher

Shanthanna $\mathrm{H}$ et al. Benefits and safety of gabapentinoids in chronic low back pain: A systematic review and meta-analysis of randomized controlled trials. PLoS Med 14(8): e1002369 